\title{
Lefschetz formulae for $p$-adic groups
}

\author{
Anton Deitmar
}

\section{Contents}

1 The trace formula 2

\begin{tabular}{|lll}
\hline 2 & The covolume of a centralizer & 7
\end{tabular}

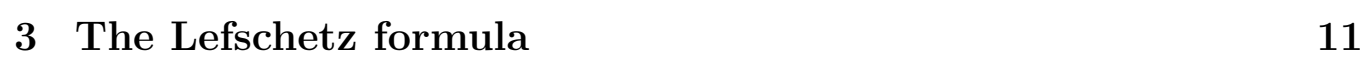




\section{Introduction}

In the paper [5] the author has extended the theory of Selberg-type zeta functions to higher rank $p$-adic groups. This extension remained incomplete insofar as within a higher rank group only elements of splitrank one were considered.

In the analogous setting of real Lie groups it emerged in recent years that the role of the Selberg zeta function in higher rank spaces is played by certain Lefschetz formulae attached to torus actions [6].

We explain this in more detail. Recall for rank one groups the relation between the Selberg zeta function and the trace formula. It is clear that the analytical properties of the zeta function are derived by means of the trace formula. Less well known is the fact that one can deduce the trace formula from the location of poles and zeros of the zeta function by evaluating a contour integral. Likewise, in the case of higher rank groups and splitrank one elements, the Selberg zeta function corresponds to a Lefschetz formula attached to the action of a minimal split torus. There is no proper analogue of the zeta function for higher rank elements, but there is a Lefschetz formula for that case, too. A special version of the Lefschetz formula in the real setting was shown in [6] and a general version in [7]. In the present paper we give the general Lefschetz formula in the $p$-adic setting.

\section{The trace formula}

Let $F$ be a nonarchimedean local field with valuation ring $\mathcal{O}$ and uniformizer $\varpi$. Denote by $G$ be a semisimple linear algebraic group over $F$. Let $K \subset G$ be a good maximal compact subgroup. Choose a parabolic subgroup $P=L N$ of $G$ with Levi component $L$. Let $A$ denote the largest split torus in the center of $L$. Then $A$ is called the split component of $P$. Let $\Phi=\Phi(G, A)$ be the root system of the pair $(G, A)$, i.e. $\Phi$ consists of all homomorphisms $\alpha: A \rightarrow \mathrm{GL}_{1}$ such that there is $X$ in the Lie algebra of $G$ with $\operatorname{Ad}(a) X=a^{\alpha} X$ for every $a \in A$. Given $\alpha$, let $\mathfrak{n}_{\alpha}$ be the Lie algebra generated by all such $X$ and let $N_{\alpha}$ be the closed subgroup of $N$ corresponding to $\mathfrak{n}_{\alpha}$. Let $\Phi^{+}=\Phi(P, A)$ be the subset of $\Phi$ consisting of all positive roots with respect to $P$. Let $\Delta \subset \Phi^{+}$ 
be the subset of simple roots. Let $A^{-} \subset A$ be the set of all $a \in A$ such that $\left|a^{\alpha}\right|<1$ for any $\alpha \in \Delta$.

There is a reductive subgroup $M$ of $L$ with compact center such that $M A$ has finite index in $L$. We can choose $M$ such that $K_{M}=M \cap K$ is a good maximal subgroup of $M$. An element $g$ of $G$ is called elliptic if it is contained in a compact torus. Let $M_{\text {ell }}$ denote the set of elliptic elements in $M$.

Let $X^{*}(A)=\operatorname{Hom}\left(A, \mathrm{GL}_{1}\right)$ be the group of all homomorphisms as algebraic groups from $A$ to $\mathrm{GL}_{1}$. This group is isomorphic to $\mathbb{Z}^{r}$ with $r=\operatorname{dim} A$. Likewise let $X_{*}(A)=\operatorname{Hom}\left(\mathrm{GL}_{1}, A\right)$. There is a natural $\mathbb{Z}$-valued pairing

$$
\begin{aligned}
X^{*}(A) \times X_{*}(A) & \rightarrow \operatorname{Hom}\left(\mathrm{GL}_{1}, \mathrm{GL}_{1}\right) \cong \mathbb{Z} \\
(\alpha, \eta) & \mapsto \alpha \circ \eta .
\end{aligned}
$$

For every root $\alpha \in \Phi(A, G) \subset X^{*}(A)$ let $\breve{\alpha} \in X_{*}(A)$ be its coroot. Then $(\alpha, \breve{\alpha})=2$. The valuation $v$ of $F$ gives a group homomorphism $\mathrm{GL}_{1}(F) \rightarrow \mathbb{Z}$. Let $A_{c}$ be the unique maximal compact subgroup of $A$. Let $\Sigma=A / A_{c}$; then $\Sigma$ is a $\mathbb{Z}$-lattice of $\operatorname{rank} r=\operatorname{dim} A$. By composing with the valuation $v$ the group $X^{*}(A)$ can be identified with

$$
\Sigma^{*}=\operatorname{Hom}(\Sigma, \mathbb{Z}) .
$$

Let

$$
\mathfrak{a}_{0}^{*}=\operatorname{Hom}(\Sigma, \mathbb{R}) \cong X^{*}(A) \otimes \mathbb{R}
$$

be the real vector space of all group homomorphisms from $\Sigma$ to $\mathbb{R}$ and let $\mathfrak{a}^{*}=\mathfrak{a}_{0}^{*} \otimes \mathbb{C}=\operatorname{Hom}(\Sigma, \mathbb{C}) \cong X^{*}(A) \otimes \mathbb{C}$. For $a \in A$ and $\lambda \in \mathfrak{a}^{*}$ let

$$
a^{\lambda}=q^{-\lambda(a)},
$$

where $q$ is the number of elements in the residue class field of $F$. In this way we get an identification

$$
\mathfrak{a}^{*} / \frac{2 \pi i}{\log q} \Sigma^{*} \cong \operatorname{Hom}\left(\Sigma, \mathbb{C}^{\times}\right) .
$$

A quasicharacter $\nu: A \rightarrow \mathbb{C}^{\times}$is called unramified if $\nu$ is trivial on $A_{c}$. The set $\operatorname{Hom}\left(\Sigma, \mathbb{C}^{\times}\right)$can be identified with the set of unramified quasicharacters on $A$. Any unramified quasicharacter $\nu$ can thus be given a unique real part $\operatorname{Re}(\nu) \in$ $\mathfrak{a}_{0}^{*}$. This definition extends to not necessarily unramified quasicharacters $\chi: A \rightarrow \mathbb{C}^{\times}$as follows. Choose a splitting $s: \Sigma \rightarrow A$ of the exact sequence

$$
1 \rightarrow A_{c} \rightarrow A \rightarrow \Sigma \rightarrow 1 \text {. }
$$


Then $\nu=\chi \circ s$ is an unramified character of $A$. Set

$$
\operatorname{Re}(\chi)=\operatorname{Re}(\nu) .
$$

This definition does not depend on the choice of the splitting $s$. For quasicharacters $\chi, \chi^{\prime}$ and $a \in A$ we will frequently write $a^{\chi}$ instead of $\chi(a)$ and $a^{\chi+\chi^{\prime}}$ instead of $\chi(a) \chi^{\prime}(a)$. Note that the absolute value satisfies $\left|a^{\chi}\right|=a^{\operatorname{Re}(\chi)}$ and that a quasicharacter $\chi$ actually is a character if and only if $\operatorname{Re}(\chi)=0$.

Let $\Delta_{P}: P \rightarrow \mathbb{R}_{+}$be the modular function of the group $P$. Then there is $\rho \in \mathfrak{a}_{0}^{*}$ such that $\Delta_{P}(a)=\left|a^{2 \rho}\right|$. For $\nu \in \mathfrak{a}^{*}$ and a root $\alpha$ let

$$
\nu_{\alpha}=(\nu, \breve{\alpha}) \in X^{*}\left(\mathrm{GL}_{1}\right) \otimes \mathbb{C} \cong \mathbb{C} .
$$

Note that $\nu \in \mathfrak{a}_{0}^{*}$ implies $\nu_{\alpha} \in \mathbb{R}$ for every $\alpha$. For $\nu \in \mathfrak{a}_{0}^{*}$ we say that $\nu$ is positive, $\nu>0$, if $\nu_{\alpha}>0$ for every positive root $\alpha$.

Example. Let $G=\mathrm{GL}_{n}(F)$ and let $\varpi_{j} \in G$ be the diagonal matrix $\varpi_{j}=$ $\operatorname{diag}(1, \ldots, 1, \varpi, 1, \ldots, 1)$ with the $\varpi$ on the $j$-th position. Let $\nu \in \mathfrak{a}^{*}$ and let

$$
\nu_{j}=\nu\left(\varpi_{j} A_{c}\right) \in \mathbb{C} .
$$

Let $\alpha$ be a root, say $\alpha\left(\operatorname{diag}\left(a_{1}, \ldots, a_{n}\right)\right)=\frac{a_{i}}{a_{j}}$. Then

$$
\nu_{\alpha}=\nu_{i}-\nu_{j} .
$$

Hence $\nu \in \mathfrak{a}_{0}^{*}$ is positive if and only if $\nu_{1}>\nu_{2}>\cdots>\nu_{n}$.

We will fix Haar-measures of $G$ and its reductive subgroups as follows. For $H \subset G$ being a torus there is a unique maximal compact subgroup $U_{H}$ which is open. Then we fix a Haar measure on $H$ such that $\operatorname{vol}\left(U_{H}\right)=1$. If $H$ is connected reductive with compact center then we choose the unique positive Haar-measure which up to sign coincides with the Euler-Poincaré measure [8. So in the latter case our measure is determined by the following property: For any discrete torsionfree cocompact subgroup $\Gamma_{H} \subset H$ we have

$$
\operatorname{vol}\left(\Gamma_{H} \backslash H\right)=(-1)^{q(H)} \chi\left(\Gamma_{H}, \mathbb{Q}\right),
$$

where $q(H)$ is the $k$-rank of the derived group $H_{\text {der }}$ and $\chi\left(\Gamma_{H}, \mathbb{Q}\right)$ the EulerPoincaré characteristic of $H^{\bullet}\left(\Gamma_{H}, \mathbb{Q}\right)$. For the applications recall that centralizers of tori in connected groups are connected [1]. 
Assume we are given a discrete subgroup $\Gamma$ of $G$ such that the quotient space $\Gamma \backslash G$ is compact. Let $\left(\omega, V_{\omega}\right)$ be a finite dimensional unitary representation of $\Gamma$ and let $L^{2}(\Gamma \backslash G, \omega)$ be the Hilbert space consisting of all measurable functions $f: G \rightarrow V_{\omega}$ such that $f(\gamma x)=\omega(\gamma) f(x)$ and $|f|$ is square integrable over $\Gamma \backslash G$ (modulo null functions). Let $R$ denote the unitary representation of $G$ on $L^{2}(\Gamma \backslash G, \omega)$ defined by right shifts, i.e. $R(g) \varphi(x)=\varphi(x g)$ for $\varphi \in$ $L^{2}(\Gamma \backslash G, \omega)$. It is known that as a $G$-representation this space splits as a topological direct sum:

$$
L^{2}(\Gamma \backslash G, \omega)=\bigoplus_{\pi \in \hat{G}} N_{\Gamma, \omega}(\pi) \pi
$$

with finite multiplicities $N_{\Gamma, \omega}(\pi)<\infty$.

Let $f$ be integrable over $G$, so $f$ is in $L^{1}(G)$. The integral

$$
R(f):=\int_{G} f(x) R(x) d x
$$

defines an operator on the Hilbert space $L^{2}(\Gamma \backslash G, \omega)$.

For $g \in G$ and $f$ any function on $G$ we define the orbital integral

$$
\mathcal{O}_{g}(f):=\int_{G_{g} \backslash G} f\left(x^{-1} g x\right) d x,
$$

whenever the integral exists. Here $G_{g}$ is the centralizer of $g$ in $G$. It is known that the group $G_{g}$ is unimodular, so we have an invariant measure on $G_{g} \backslash G$.

A function $f$ on $G$ or any of its closed subgroups is called smooth if it is locally constant. It is called uniformly smooth if there is an open subgroup $U$ of $G$ such that $f$ factors over $U \backslash G / U$. This is in particular the case if $f$ is smooth and compactly supported.

Proposition 1.1 (Trace formula) Let $f$ be integrable and uniformly smooth, then we have

$$
\sum_{\pi \in \hat{G}} N_{\Gamma, \omega}(\pi) \operatorname{tr} \pi(f)=\sum_{[\gamma]} \operatorname{tr} \omega(\gamma) \operatorname{vol}\left(\Gamma_{\gamma} \backslash G_{\gamma}\right) \mathcal{O}_{\gamma}(f),
$$

where the sum on the right hand side runs over the set of $\Gamma$-conjugacy classes $[\gamma]$ in $\Gamma$ and $\Gamma_{\gamma}$ denotes the centralizer of $\gamma$ in $\Gamma$. Both sides converge absolutely and the left hand side actually is a finite sum. 
Proof: At first fix a fundamental domain $\mathcal{F}$ for $\Gamma \backslash G$ and let $\varphi \in L^{2}(\Gamma \backslash G, \omega)$, then

$$
\begin{aligned}
R(f) & =\int_{G} f(y) \varphi(x y) d y \\
& =\int_{G} f\left(x^{-1} y\right) \varphi(y) d y \\
& =\sum_{\gamma \in \Gamma} \int_{\mathcal{F}} f\left(x^{-1} \gamma y\right) \varphi(\gamma y) d y \\
& =\int_{\Gamma \backslash G}\left(\sum_{\gamma \in \Gamma} f\left(x^{-1} \gamma y\right) \omega(\gamma)\right) \varphi(y) d y
\end{aligned}
$$

We want to show that the sum $\sum_{\gamma \in \Gamma} f\left(x^{-1} \gamma y\right) \omega(\gamma)$ converges in $\operatorname{End}\left(V_{\omega}\right)$ absolutely and uniformly in $x$ and $y$. Since $y$ can be replaced by $\gamma y, \gamma \in \Gamma$ and since $\omega$ is unitary, we only have to show the convergence of $\sum_{\gamma \in \Gamma}\left|f\left(x^{-1} \gamma y\right)\right|$ locally uniformly in $y$. Let $\gamma$ and $\tau$ be in $\Gamma$ and assume that $x^{-1} \gamma y$ and $x^{-1} \tau y$ lie in the same class in $G / U$. Then it follows $\tau y U \cap \gamma y U \neq \emptyset$ so with $V=y U y^{-1}$ we have $\gamma^{-1} \tau V \cap V \neq \emptyset$. It is clear that $V$ depends on $y$ only up to $U$ so to show locally uniform convergence in $y$ it suffices to fix $V$. Since $V$ is compact also $V^{2}=\left\{v v^{\prime} \mid v, v^{\prime} \in V\right\}$ is compact and so $\Gamma \cap V^{2}$ is finite. This implies that there are only finitely many $\gamma \in \Gamma$ with $\gamma V \cap V \neq \emptyset$. Hence the map $\Gamma \rightarrow G / U, \gamma \mapsto x^{-1} \gamma y U$ is finite to one with fibers having $\leq n$ elements for some natural number $n$. For $y$ fixed modulo $U$ we get

$$
\begin{aligned}
\sum_{\gamma \in \Gamma}\left|f\left(x^{-1} \gamma y\right)\right| & \leq n \int_{G / U}|f(x)| d x \\
& =\frac{n}{\operatorname{vol}(U)}\|f\|^{1} .
\end{aligned}
$$

We have shown the uniform convergence of the sum

$$
k_{f}(x, y)=\sum_{\gamma \in \Gamma} f\left(x^{-1} \gamma y\right) \omega(\gamma) .
$$

Observe that $R(f)$ factors over $L^{2}(\Gamma \backslash G, \omega)^{U}=L^{2}(\Gamma \backslash G / U, \omega)$, which is finite dimensional since $\Gamma \backslash G / U$ is a finite set. So $R(f)$ acts on a finite dimensional 
space and $k_{f}(x, y)$ is the matrix of this operator. We infer that $R(f)$ is of trace class, its trace equals

$$
\sum_{\pi \in \hat{G}} N_{\Gamma, \omega}(\pi) \operatorname{tr} \pi(f),
$$

and the sum is finite. Further, since $k_{f}(x, y)$ is the matrix of $R(f)$ this trace also equals

$$
\begin{aligned}
\int_{\Gamma \backslash G} \operatorname{tr} k_{f}(x, x) d x & =\sum_{\gamma \in \Gamma} \int_{\mathcal{F}} f\left(x^{-1} \gamma x\right) d x \operatorname{tr} \omega(\gamma) \\
& =\sum_{[\gamma]} \sum_{\sigma \in \Gamma_{\gamma} \backslash \Gamma} \int_{\mathcal{F}} f\left((\sigma x)^{-1} \gamma(\sigma x)\right) d x \operatorname{tr} \omega(\gamma) \\
& =\sum_{[\gamma]} \int_{\Gamma_{\gamma} \backslash G} f\left(x^{-1} \gamma x\right) d x \operatorname{tr} \omega(\gamma) \\
& =\sum_{[\gamma]} \operatorname{vol}\left(\Gamma_{\gamma} \backslash G_{\gamma}\right) \int_{G_{\gamma} \backslash G} f\left(x^{-1} \gamma x\right) d x \operatorname{tr} \omega(\gamma)
\end{aligned}
$$

\section{The covolume of a centralizer}

Suppose $\gamma \in \Gamma$ is $G$-conjugate to some $a_{\gamma} m_{\gamma} \in A^{+} M_{\text {ell }}$. We want to compute the covolume

$$
\operatorname{vol}\left(\Gamma_{\gamma} \backslash G_{\gamma}\right)
$$

An element $x$ of $G$ is called neat if for every representation $\rho: G \rightarrow G L_{n}(F)$ of $G$ the matrix $\rho(x)$ has no eigenvalue which is a root of unity different from 1. A subset $A$ of $G$ is called neat if each element of it is. Every arithmetic $\Gamma$ has a finite index subgroup which is neat [2].

Lemma 2.1 Let $x \in G$ be neat and semisimple. Let $G_{x}$ denote its centralizer in $G$. Then for every $k \in \mathbb{N}$ we have $G_{x}=G_{x^{k}}$. 
Proof: Since $G$ is linear algebraic it is a subgroup of some $H=\operatorname{GL}_{n}(\bar{F})$, where $\bar{F}$ is an algebraic closure of $F$. If we can show the claim for $H$ then it follows for $G$ as well since $G_{x}=H_{x} \cap G$. In $H$ we can assume $x$ to be a diagonal matrix. Since $x$ is neat this implies the claim.

We suppose that $\Gamma$ is neat. This implies that for any $\gamma \in \Gamma$ the Zariski closure of the group generated by $\gamma$ is a torus. It then follows that $G_{\gamma}$ is a connected reductive group [1].

An element $\gamma \in \Gamma$ is called primitive if $\gamma=\sigma^{n}$ with $\sigma \in \Gamma$ and $n \in \mathbb{N}$ implies $n=1$. It is a property of discrete cocompact torsion free subgroups $\Gamma$ of $G$ that every $\gamma \in \Gamma, \gamma \neq 1$ is a positive power of a unique primitive element. In other words, given a nontrivial $\gamma \in \Gamma$ there exists a unique primitive $\gamma_{0}$ and a unique $\mu(\gamma) \in \mathbb{N}$ such that

$$
\gamma=\gamma_{0}^{\mu(\gamma)}
$$

Let $\Sigma$ be a group of finite cohomological dimension $c d(\Sigma)$ over $\mathbb{Q}$. We write

$$
\chi(\Sigma)=\chi(\Sigma, \mathbb{Q}):=\sum_{p=0}^{c d(\Sigma)}(-1)^{p} \operatorname{dim} H^{p}(\Sigma, \mathbb{Q}),
$$

for the Euler-Poincaré characteristic. We also define the higher Euler characteristic as

$$
\chi_{r}(\Sigma)=\chi_{r}(\Sigma, \mathbb{Q}):=\sum_{p=0}^{c d(\Sigma)}(-1)^{p+r}\left(\begin{array}{l}
p \\
r
\end{array}\right) \operatorname{dim} H^{p}(\Sigma, \mathbb{Q}),
$$

for $r=1,2,3, \ldots$ It is known that $\Gamma$ has finite cohomological dimension over Q.

We denote by $\mathcal{E}_{P}(\Gamma)$ the set of all conjugacy classes $[\gamma]$ in $\gamma$ such that $\gamma$ is in $G$ conjugate to an element $a_{\gamma} m_{\gamma} \in A M$, where $m_{\gamma}$ is elliptic and $a_{\gamma} \in A^{-}$.

Let $\gamma \in \mathcal{E}_{P}(\Gamma)$. To simplify the notation let's assume that $\gamma=a_{\gamma} m_{\gamma} \in$ $A^{-} M_{\text {ell }}$. Let $C_{\gamma}$ be the connected component of the center of $G_{\gamma}$ then $C_{\gamma}=$ $A B_{\gamma}$, where $B_{\gamma}$ is the connected center of $M_{m_{\gamma}}$ the latter group will also be written as $M_{\gamma}$. Let $M_{\gamma}^{\text {der }}$ be the derived group of $M_{\gamma}$. Then $M_{\gamma}=M_{\gamma}^{\text {der }} B_{\gamma}$. 
Lemma 2.2 $B_{\gamma}$ is compact.

Proof: Since $m_{\gamma}$ is elliptic there is a compact Cartan subgroup $T$ of $M$ containing $m_{\gamma}$. Since $M$ modulo its center is a connected semisimple linear algebraic group it follows that $T$ is a torus and therefore abelian. Therefore $T \subset M_{m_{\gamma}}$. Let $b \in B_{\gamma}$. Then $b$ commutes with every $t \in T$, therefore $b$ lies in the centralizer of $T$ in $M$ which equals $T$. So we have shown $B_{\gamma} \subset T$.

Let $\Gamma_{\gamma, A}=A \cap \Gamma_{\gamma} B_{\gamma}$ and $\Gamma_{\gamma, M}=M_{\gamma}^{\text {der }} \cap \Gamma_{\gamma} A B_{\gamma}$. Similar to the proof of Lemma 3.3 of [9] one shows that $\Gamma_{\gamma, A}$ and $\Gamma_{\gamma, M}$ are discrete cocompact subgroups of $A$ and $M_{\gamma}^{\text {der }}$ resp. Let

$$
\lambda_{\gamma} \stackrel{\text { def }}{=} \operatorname{vol}\left(\Gamma_{\gamma, A} \backslash A\right)
$$

Proposition 2.3 Assume $\Gamma$ neat and let $\gamma \in \Gamma$ be $G$-conjugate to an element of $A^{+} M_{\text {ell }}$. Then we get

$$
\operatorname{vol}\left(\Gamma_{\gamma} \backslash G_{\gamma}\right)=\lambda_{\gamma}(-1)^{q(G)+r} \chi_{r}\left(\Gamma_{\gamma}\right)
$$

where $r=\operatorname{dim} A$.

Proof: We normalize the volume of $B_{\gamma}$ to be 1 . Then

$$
\begin{aligned}
\operatorname{vol}\left(\Gamma_{\gamma} \backslash G_{\gamma}\right) & =\operatorname{vol}\left(\Gamma_{\gamma} \backslash A M_{\gamma}\right) \\
& =\operatorname{vol}\left(\Gamma_{\gamma} B_{\gamma} \backslash A M_{\gamma}\right)
\end{aligned}
$$

The space $\Gamma_{\gamma} B_{\gamma} \backslash A M_{\gamma}$ is the total space of a fibration with fibre $\Gamma_{\gamma, A} \backslash A$ and base space $\Gamma_{\gamma} A B_{\gamma} \backslash M_{\gamma} A \cong \Gamma_{\gamma, M} \backslash M_{\gamma}^{\text {der }}$. Hence

$$
\operatorname{vol}\left(\Gamma_{\gamma} B_{\gamma} \backslash A M_{\gamma}\right)=\operatorname{vol}\left(\Gamma_{\gamma, A} \backslash A\right) \operatorname{vol}\left(\Gamma_{\gamma, M} \backslash M_{\gamma}^{\text {der }}\right) .
$$

Since $\lambda_{\gamma}=\operatorname{vol}\left(\Gamma_{\gamma, A} \backslash A\right)$ it remains to show

$$
\operatorname{vol}\left(\Gamma_{\gamma, M} \backslash M_{\gamma}^{\text {der }}\right)=(-1)^{r} \chi_{r}\left(\Gamma_{\gamma}\right)
$$

We know that

$$
\operatorname{vol}\left(\Gamma_{\gamma, M} \backslash M_{\gamma}^{d e r}\right)=(-1)^{q\left(M_{\gamma}\right)} \chi\left(\Gamma_{\gamma, M}\right)=(-1)^{q(G)+r} \chi\left(\Gamma_{\gamma, M}\right) .
$$

So it remains to show that $\chi\left(\Gamma_{\gamma, M}\right)=\chi_{r}\left(\Gamma_{\gamma}\right)$. The group $\Gamma_{\gamma, M}$ is isomorphic to $\Gamma_{\gamma} / \Sigma$, where $\Sigma=\Gamma \cap A B_{\gamma}$ is isomorphic to $\mathbb{Z}^{r}$. So the proposition follows from the next Lemma. 
Lemma 2.4 Let $\Gamma, \Lambda$ be of finite cohomological dimension over $\mathbb{Q}$. Let $C_{r}$ be a group isomorphic to $\mathbb{Z}^{r}$ and assume there is an exact sequence

$$
1 \rightarrow C_{r} \rightarrow \Gamma \rightarrow \Lambda \rightarrow 1 \text {. }
$$

Assume that $C_{r}$ is central in $\Gamma$. Then

$$
\chi(\Lambda, \mathbb{Q})=\chi_{r}(\Gamma, \mathbb{Q}) .
$$

Proof: We first consider the case $r=1$. In this case we want to prove for every $r$,

$$
\chi_{r-1}(\Lambda, \mathbb{Q})=\chi_{r}(\Gamma, \mathbb{Q}) .
$$

For this consider the Hochschild-Serre spectral sequence:

$$
E_{2}^{p, q}=H^{p}\left(\Lambda, H^{q}\left(C_{1}, \mathbb{Q}\right)\right)
$$

which abuts to

$$
H^{p+q}(\Gamma, \mathbb{Q})
$$

Since $C_{1} \cong \mathbb{Z}$ it follows

$$
H^{q}\left(C_{1}, \mathbb{Q}\right)= \begin{cases}\mathbb{Q} & \text { if } q=0,1 \\ 0 & \text { else. }\end{cases}
$$

Since $C_{1}$ is infinite cyclic and central it is an exercise to see that the spectral sequence degenerates at $E_{2}$. Therefore,

$$
\begin{aligned}
\chi_{r}(\Gamma)= & \sum_{j \geq 0}(-1)^{j+r}\left(\begin{array}{l}
j \\
r
\end{array}\right) \operatorname{dim} H^{j}(\Gamma) \\
= & \sum_{j \geq r}(-1)^{j+r}\left(\begin{array}{l}
j \\
r
\end{array}\right)\left(\operatorname{dim} H^{j}(\Lambda)+\operatorname{dim} H^{j-1}(\Lambda)\right. \\
= & \sum_{j \geq r}(-1)^{j+r}\left(\begin{array}{l}
j \\
r
\end{array}\right) \operatorname{dim} H^{j}(\Lambda) \\
& -\sum_{j \geq r-1}(-1)^{j+r}\left(\begin{array}{c}
j+1 \\
r
\end{array}\right) \operatorname{dim} H^{j}(\Lambda) .
\end{aligned}
$$

Now replace $\left(\begin{array}{c}j+1 \\ r\end{array}\right)$ by $\left(\begin{array}{c}j \\ r\end{array}\right)+\left(\begin{array}{c}j \\ r-1\end{array}\right)$ to get the claim. For the general case write $C_{r}=C_{1} \oplus C^{1}$, where $C_{1}$ is cyclic and $C^{1} \cong \mathbb{Z}^{r-1}$. Apply the above to $C_{1}$ and iterate this to get the lemma and hence the proposition. 


\section{The Lefschetz formula}

For a representation $\pi$ of $G$ let $\pi^{\infty}$ denote the subrepresentation of smooth vectors, ie $\pi^{\infty}$ is the representation on the space $\bigcup_{H \subset G} \pi^{H}$, where $H$ ranges over the set of all open subgroups of $G$. Further let $\pi_{N}$ denote the Jacquet module of $\pi$. By definition $\pi_{N}$ is the largest quotient $M A N$-module of $\pi^{\infty}$ on which $N$ acts trivially. One can achieve this by factoring out the vector subspace consisting of all vectors of the form $v-\pi(n) v$ for $v \in \pi^{\infty}, n \in N$. It is known that if $\pi$ is an irreducible admissible representation, then $\pi_{N}$ is a admissible $M A$-module of finite length. For a smooth $M$-module $V$ let $H_{c}^{\bullet}(M, V)$ denote the continuous cohomology with coefficients in $V$ as in 3 .

Theorem 3.1 (Lefschetz Formula)

Let $\Gamma$ be a neat discrete cocompact subgroup of $G$. Let $\varphi$ be a uniformly smooth function on $A$ with support in $A^{-}$. Suppose that the function $a \mapsto \varphi(a)\left|a^{-2 \rho}\right|$ is integrable on $A$. Let $\sigma$ be a finite dimensional representation of $M$. Let $q$ be the F-splitrank of $G$ and $r=\operatorname{dim} A$.Then

$$
\sum_{\pi \in \hat{G}} N_{\Gamma, \omega}(\pi) \sum_{q=0}^{\operatorname{dim} M}(-1)^{a} \int_{A^{-}} \varphi(a) \operatorname{tr}\left(a \mid H_{c}^{q}\left(M, \pi_{N} \otimes \sigma\right)\right) d a
$$

equals

$$
(-1)^{q+r} \sum_{[\gamma] \in \mathcal{E}_{P}(\Gamma)} \lambda_{\gamma} \chi_{r}\left(\Gamma_{\gamma}\right) \operatorname{tr} \omega(\gamma) \operatorname{tr} \sigma\left(m_{\gamma}\right) \varphi\left(a_{\gamma}\right)\left|a_{\gamma}^{2 \rho}\right| .
$$

Both outer sums converge absolutely and the sum over $\pi \in \hat{G}$ actually is a finite sum, ie, the summand is zero for all but finitely many $\pi$. For a given compact open subgroup $U$ of $A$ both sides represent a continuous linear functional on the space of all functions $\varphi$ as above which factor over $A / U$, where this space is equipped with the norm $\|\varphi\|=\int_{A}\left|\varphi(a) \| a^{-2 \rho}\right| d a$.

Let $A^{*}$ denote the set of all continuous group homomorphisms $\lambda: A \rightarrow \mathbb{C}^{\times}$. For $\lambda \in A^{*}$ and an $A$-module $V$ let $V_{\lambda}$ denote the generalized $\lambda$-eigenspace, ie,

$$
V_{\lambda} \stackrel{\text { def }}{=} \bigcup_{k=1}^{\infty}\left\{v \in V \mid(a-\lambda(a))^{k} v=0 \forall a \in A\right\} .
$$


Then

$$
\int_{A^{-}} \varphi(a) \operatorname{tr}\left(a \mid H_{c}^{q}\left(M, \pi_{N} \otimes \sigma\right)\right) d a=\sum_{\lambda \in A^{*}} \operatorname{dim} H_{c}^{q}\left(M, \pi_{N} \otimes \sigma\right)_{\lambda} \int_{A^{-}} \varphi(a \lambda(a d a .
$$

For $\lambda \in A^{*}$ define

$$
m_{\lambda}^{\sigma, \omega} \stackrel{\operatorname{def}}{=} \sum_{\pi \in \hat{G}} N_{\Gamma, \omega}(\pi) \sum_{q=0}^{\operatorname{dim} M}(-1)^{q} \operatorname{dim} H_{c}^{q}\left(M, \pi_{N} \otimes \sigma\right)_{\lambda}
$$

The sum is always finite.

On the other hand, for $[\gamma] \in \mathcal{E}_{P}(\Gamma)$ let

$$
c_{\gamma} \stackrel{\text { def }}{=} \lambda_{\gamma} \chi_{r}\left(\Gamma_{\gamma}\right)\left|a_{\gamma}^{2 \rho}\right|
$$

Then the Theorem is equivalent to the following Corollary.

Corollary 3.2 (Lefschetz Formula)

As an identity of distributions on $A^{-}$we have

$$
\sum_{\lambda \in A^{*}} m_{\lambda}^{\sigma, \omega} \lambda=\sum_{[\gamma] \in \mathcal{E}_{P}(\Gamma)} c_{\gamma} \operatorname{tr} \omega(\gamma) \operatorname{tr} \sigma\left(m_{\gamma}\right) \delta_{a_{\gamma}}
$$

Proof of the Theorem: Let $f_{E P}$ be an Euler-Poincaré function on $M$ which is $K_{M}$-central [8]. For $m \in M$ regular we have

$$
\mathcal{O}_{m}^{M}\left(f_{E P}\right)= \begin{cases}1 & m \text { elliptic } \\ 0 & \text { otherwise }\end{cases}
$$

For $g \in G$ and a finite dimensional $F$ vector space $V$ on which $g$ acts linearly let $E(g \mid V)$ be the set of all absolute values $|\mu|$, where $\mu$ ranges over the eigenvalues of $g$ in the algebraic closure $\bar{F}$ of $F$. Let $\lambda_{\min }(g \mid V)$ denote the minimum and $\lambda_{\max }(v \mid V)$ the maximum of $E(g \mid V)$. For am $\in A M$ define

$$
\lambda(a m) \stackrel{\text { def }}{=} \frac{\lambda_{\min }(a \mid \overline{\mathfrak{n}})}{\lambda_{\max }(m \mid \mathfrak{g})^{2}}
$$


Note that $\lambda_{\max }(m \mid \mathfrak{g})$ is always $\geq 1$ and that $\lambda_{\max }(m \mid \mathfrak{g}) \lambda_{\min }(m \mid \mathfrak{g})=1$. We will consider the set

$$
(A M)^{\sim}:=\{a m \in A M \mid \lambda(a m)>1\} .
$$

Let $M_{\text {ell }}$ denote the set of elliptic elements in $M$.

Lemma 3.3 The set $(A M)^{\sim}$ has the following properties:

1. $A^{-} M_{\text {ell }} \subset(A M)^{\sim}$

2. $a m \in(A M)^{\sim} \Rightarrow a \in A^{-}$

3. $a m, a^{\prime} m^{\prime} \in(A M)^{\sim}, g \in G$ with $a^{\prime} m^{\prime}=g a m g^{-1} \Rightarrow a=a^{\prime}, g \in A M$.

Proof: The first two are immediate. For the third let $a m, a^{\prime} m^{\prime} \in(A M)^{\sim}$ and $g \in G$ with $a^{\prime} m^{\prime}=g a m g^{-1}$. Observe that by the definition of $(A M)^{\sim}$ we have

$$
\begin{aligned}
\lambda_{\min }(\operatorname{am} \mid \overline{\mathfrak{n}}) & \geq \lambda_{\min }(a \mid \overline{\mathfrak{n}}) \lambda_{\min }(m \mid \mathfrak{g}) \\
& >\lambda_{\max }(m \mid \mathfrak{g})^{2} \lambda_{\min }(m \mid \mathfrak{g}) \\
& =\lambda_{\max }(m \mid \mathfrak{g}) \\
& \geq \lambda_{\max }(m \mid \mathfrak{a}+\mathfrak{m}+\mathfrak{n}) \\
& \geq \lambda_{\max }(a m \mid \mathfrak{a}+\mathfrak{m}+\mathfrak{n})
\end{aligned}
$$

that is, any eigenvalue of $a m$ on $\overline{\mathfrak{n}}$ is strictly bigger than any eigenvalue on $\mathfrak{a}+\mathfrak{m}+\mathfrak{n}$. Since $\mathfrak{g}=\mathfrak{a}+\mathfrak{m}+\mathfrak{n}+\overline{\mathfrak{n}}$ and the same holds for $a^{\prime} m^{\prime}$, which has the same eigenvalues as $a m$, we infer that $\operatorname{Ad}(g) \overline{\mathfrak{n}}=\overline{\mathfrak{n}}$. So $g$ lies in the normalizer of $\overline{\mathfrak{n}}$, which is $\bar{P}=M A \bar{N}=\bar{N} A M$. Now suppose $g=n m_{1} a_{1}$ and $\hat{m}=m_{1} m m_{1}^{-1}$ then

$$
\operatorname{gamg}^{-1}=n a \hat{m} n^{-1}=a \hat{m}(a \hat{m})^{-1} n(a \hat{m}) n^{-1} .
$$

Since this lies in $A M$ we have $(a \hat{m})^{-1} n(a \hat{m})=n$ which since $a m \in(A M)^{\sim}$ implies $n=1$. The lemma is proven.

Let $G$ act on itself by conjugation, write $g \cdot x=g x g^{-1}$, write $G \cdot x$ for the orbit, so $G . x=\left\{g x g^{-1} \mid g \in G\right\}$ as well as $G . S=\left\{g s g^{-1} \mid s \in S, g \in G\right\}$ for any subset $S$ of $G$. 
Fix a smooth function $\eta$ on $N$ which has compact support, is positive, invariant under $K_{M}$ and satisfies $\int_{N} \eta(n) d n=1$. Extend the function $\varphi$ from $A^{-}$to a conjugation invariant smooth function $\tilde{\varphi}$ on $A M$ such that $\tilde{\varphi}(a m)=\varphi(a)$ whenever $m$ is elliptic and such that there is a compact subset $C \subset A^{-}$such that $\tilde{\varphi}$ is supported in $C M \cap(A M)^{\sim}$. It follows that the function

$$
a m \mapsto f_{E P}(m) \operatorname{tr} \sigma(m) \tilde{\varphi}(a m)\left|a^{2 \rho}\right|
$$

is smooth and integrable on $A M$. Given these data let $f=f_{\eta, \tau, \varphi}: H \rightarrow \mathbb{C}$ be defined by

$$
f\left(k n m a(k n)^{-1}\right):=\eta(n) f_{E P}(m) \operatorname{tr} \sigma(m) \tilde{\varphi}(a m)\left|a^{2 \rho}\right|,
$$

for $k \in K, n \in N, m \in M, a \in \overline{A^{-}}$. Further $f(x)=0$ if $x$ is not in $G$. $(A M)^{\sim}$.

Lemma 3.4 The function $f$ is well defined.

Proof: By the decomposition $G=K P=K N M A$ every element $x \in$ $G$. $(A M)^{\sim}$ can be written in the form $k n m a(k n)^{-1}$. Now suppose two such representations coincide, that is

$$
k n m a(k n)^{-1}=k^{\prime} n^{\prime} m^{\prime} a^{\prime}\left(k^{\prime} n^{\prime}\right)^{-1}
$$

then by Lemma 3.3 we get $\left(n^{\prime}\right)^{-1}\left(k^{\prime}\right)^{-1} k n \in M A$, or $\left(k^{\prime}\right)^{-1} k \in n^{\prime} M A n^{-1} \subset$ $M A N$, hence $\left(k^{\prime}\right)^{-1} k \in K \cap M A N=K \cap M=K_{M}$. Write $\left(k^{\prime}\right)^{-1} k=k_{M}$ and $n^{\prime \prime}=k_{M} n k_{M}^{-1}$, then it follows

$$
n^{\prime \prime} k_{M} m k_{M}^{-1} a\left(n^{\prime \prime}\right)^{-1}=n^{\prime} m^{\prime} a^{\prime}\left(n^{\prime}\right)^{-1} .
$$

Again by Lemma 3.3 we conclude $\left(n^{\prime}\right)^{-1} n^{\prime \prime} \in M A$, hence $n^{\prime}=n^{\prime \prime}$ and so

$$
k_{M} m k_{M}^{-1} a=m^{\prime} a^{\prime},
$$

which implies the well-definedness of $f$.

We will plug $f$ into the trace formula. For the geometric side let $\gamma \in \Gamma$. We have to calculate the orbital integral:

$$
\mathcal{O}_{\gamma}(f)=\int_{G_{\gamma} \backslash G} f\left(x^{-1} \gamma x\right) d x .
$$


by the definition of $f$ it follows that $\mathcal{O}_{\gamma}(f)=0$ if $\gamma \notin G$. $(A M)^{\sim}$. It remains to compute $\mathcal{O}_{a m}(f)$ for $a m \in(A M)^{\sim}$. Again by the definition of $f$ it follows

$$
\begin{aligned}
\mathcal{O}_{a m}(f) & =\mathcal{O}_{m}^{M}\left(f_{E P}\right) \operatorname{tr} \sigma(m) \tilde{\varphi}(a m)\left|a^{2 \rho}\right| \\
& = \begin{cases}\operatorname{tr} \sigma(m) \varphi(a)\left|a^{2 \rho}\right| & \text { if } m \text { is elliptic } \\
0 & \text { otherwise }\end{cases}
\end{aligned}
$$

Here $\mathcal{O}_{m}^{M}$ denotes the orbital integral in the group $M$. Recall that Proposition 2.3 says

$$
\operatorname{vol}\left(\Gamma_{\gamma} \backslash G_{\gamma}\right)=(-1)^{q(G)+r} \lambda_{\gamma} \chi_{r}\left(\Gamma_{\gamma}\right),
$$

so that for $\gamma \in \mathcal{E}_{P}(\Gamma)$,

$$
\operatorname{vol}\left(\Gamma_{\gamma} \backslash G_{\gamma}\right) \mathcal{O}_{\gamma}(f)=(-1)^{q(G+r} \lambda_{\gamma} \chi_{r}\left(\Gamma_{\gamma}\right) \operatorname{tr} \sigma\left(m_{\gamma}\right) \varphi\left(a_{\gamma}\right)\left|a_{\gamma}^{2 \rho}\right| .
$$

To compute the spectral side let $\pi \in \hat{G}$. We want to compute $\operatorname{tr} \pi(f)$. Let $\Theta_{\pi}^{G}$ be the locally integrable conjugation invariant function on $G$ such that

$$
\operatorname{tr} \pi(f)=\int_{G} f(x) \Theta_{\pi}^{G}(x) d x
$$

This function $\Theta_{\pi}$ is called the character of $\pi$. It is known that the Jacquet module $\pi_{N}$ is a finitely generated admissible module for the group $M A$ and therefore it has a character $\Theta_{\pi_{N}}^{M A}$. In [4] it is shown that

$$
\Theta_{\pi}(a m)=\Theta_{\pi_{N}}^{M A}(m a)
$$

for $m a \in A^{-} M_{e l l}$.

Let $h$ be a function in $L^{1}(G)$ which is supported in the set $G . M A$. Comparing invariant differential forms as in the proof of the Weyl integration formula one gets that the integral $\int_{G} h(x) d x$ equals

$$
\frac{1}{|W(G, A)|} \int_{A} \int_{M} \int_{G / A M} h\left(y a m y^{-1}\right)|\operatorname{det}(1-a m \mid \mathfrak{n}+\overline{\mathfrak{n}})| d y d a d m
$$

where $W(G, A)$ is the Weyl group of $A$ in $G$. 
For $a \in A^{-}$and $m \in M_{\text {ell }}$ every eigenvalue of $a m$ on $\mathfrak{n}$ is of absolute value $<1$ and $>1$ on $\overline{\mathfrak{n}}$. By the ultrametric ptoperty this implies

$$
\begin{aligned}
|\operatorname{det}(1-a m \mid \mathfrak{n}+\overline{\mathfrak{n}})| & =|\operatorname{det}(1-a m \mid \overline{\mathfrak{n}})| \\
& =|\operatorname{det}(\operatorname{am} \mid \overline{\mathfrak{n}})| \\
& =|\operatorname{det}(a \mid \overline{\mathfrak{n}})| \\
& =\left|a^{-2 \rho}\right|
\end{aligned}
$$

We apply this to $h(x)=f(x) \Theta_{\pi}^{G}(x)$ and use conjugation invariance of $\Theta_{\pi}^{G}$ to get that $\operatorname{tr} \pi(f)$ equals

$$
\frac{1}{|W(G, A)|} \int_{A M} f_{E P}(m) \operatorname{tr} \sigma(m) \tilde{\varphi}(a m) \Theta_{\pi_{N}}^{M}(a m) d a d m
$$

which is the same as

$$
\int_{A^{-} M} f_{E P}(m) \operatorname{tr} \sigma(m) \tilde{\varphi}(a m) \Theta_{\pi_{N}}^{M}(a m) d a d m .
$$

We recall the Weyl integration formula for $M$. Let $\left(H_{j}\right)_{j}$ be a maximal family of pairwise non-conjugate Cartan subgroups of $M$. Let $W_{j}$ be the Weyl group of $H_{j}$ in $M$. For $h \in H_{j}$ let $D_{j}(h)=\operatorname{det}\left(1-h \mid \mathfrak{m} / \mathfrak{h}_{j}\right)$, where $\mathfrak{m}$ and $\mathfrak{h}_{j}$ are the Lie algebras of $M$ and $H_{j}$ resp. Then, for every $h \in L^{1}(M)$,

$$
\begin{aligned}
\int_{M} h(m) d m & =\sum_{j} \frac{1}{\left|W_{j}\right|} \int_{H_{j}^{r e g}} \int_{M / H_{j}} h\left(m x m^{-1}\right) D_{j}(x) d m d x \\
& =\sum_{j} \frac{1}{\left|W_{j}\right|} \int_{H_{j}} \mathcal{O}_{x}^{M}(h) d x
\end{aligned}
$$

where $H_{j}^{r e g}$ is the set of $x \in H_{j}$ which are regular in $M$. We fix $a \in A^{-}$and apply this to $h(m)=f_{E P}(m) \tilde{\varphi}(a m) \operatorname{tr} \sigma(m) \Theta_{\pi_{N}}^{M}(a m)$. Since $\tilde{\varphi}$ is conjugation invariant we get for $x \in H_{j}^{r e g}$,

$$
\mathcal{O}_{x}^{M}(h)=\mathcal{O}_{x}^{M}\left(f_{E P}\right) \tilde{\varphi}(a m) \operatorname{tr} \sigma(m) \Theta_{\pi_{N}}^{A M}(a x) .
$$

This is non-zero only if $x$ is elliptic. If $x$ is elliptic, then $\tilde{\varphi}(a x)$ equals $\varphi(a)$. So we can replace $\tilde{\varphi}(a x)$ by $\varphi(a)$ throughout. Thus $\operatorname{tr} \pi(f)$ equals

$$
\int_{A^{-} M} f_{E P}(m) \varphi(a) \operatorname{tr} \sigma(m) \Theta_{\pi_{N}}^{M}(a m) d a d m .
$$


The trace $\operatorname{tr} \pi(f)$ therefore equals

$$
\int_{A^{-} M} f_{E P}(m) \varphi(a) \Theta_{\pi_{N} \otimes \sigma}(a m) d a d m .
$$

We write $H_{c}^{\bullet}(M, V)$ for the continuous cohomology of $M$ with coefficients in the $M$-module $V$. By Theorem 2 in [8],

$$
\operatorname{tr}\left(\pi_{N} \otimes \sigma\right)\left(f_{E P}\right)=\sum_{q=0}^{\operatorname{dim} M}(-1)^{q} \operatorname{dim} H_{c}^{q}\left(M, \pi_{N} \otimes \sigma\right) .
$$

The cohomology groups $H_{c}^{q}\left(M, \pi_{N} \otimes \sigma\right)$ are finite dimensional $A$-modules and

$$
\operatorname{tr} \pi(f)=\sum_{q=0}^{\operatorname{dim} M}(-1)^{q} \int_{A^{-}} \operatorname{tr}\left(a \mid H_{c}^{q}\left(M, \pi_{N} \otimes \sigma\right)\right) \varphi(a) d a .
$$

The Lefschetz Theorem follows. 


\section{References}

[1] Borel, A.: Linear Algebraic Groups. W.A.Benjamin Inc. New York 1969.

[2] Borel, A.: Introduction aux groupes arithmétiques. Hermann, Paris 1969.

[3] Borel, A.; Wallach,N.: Continuous Cohomology, Discrete Groups, and Representations of Reductive Groups. Ann. Math. Stud. 94, Princeton 1980.

[4] Casselman, W.: Characters and Jacquet Modules. Math. Ann. 230, 101-105 (1977).

[5] Deitmar, A.: Geometric zeta-functions on p-adic groups. Math. Japon. 47, No. 1, 1-17 (1998).

[6] Deitmar, A.: A prime geodesic theorem for higher rank spaces. Geometric and Functional Analysis 14, 1238-1266 (2004).

[7] Deitmar, A.: A Lefschetz formula for higher rank preprint

[8] Kottwitz, R.: Tamagawa Numbers. Ann. Math. 127, 629-646 (1988).

[9] Wolf, J.: Discrete groups, symmetric spaces and global holonomy. Am. J. Math. 84, 527-542 (1962).

University of Exeter, Mathematics, Exeter EX4 4QE, Devon, UK a.h.j.deitmar@ex.ac.uk 